\title{
Characterizing the VHE emission of LS I +61 303 using VERITAS observations
}

\author{
D.B Kieda ${ }^{* 1}$ for the VERITAS Collaboration ${ }^{\dagger}$ \\ ${ }^{1}$ Department of Physics and Astronomy University of Utah, Salt Lake City, UT 84112, USA \\ e-mail: dave.kiedalutah.edu
}

\begin{abstract}
The TeV gamma-ray binary LS I +61303 , approximately $2 \mathrm{kpc}$ from Earth, consists of a low mass compact object in an eccentric orbit around a massive Be star. LS I +61 303 exhibits modulated VHE gamma-ray emission around its 26.5 days orbit, with strongest $\mathrm{TeV}$ emission during its apastron passage (orbital phases $\varphi=0.55-0.65$ ). Multiple flaring episodes with nightly flux variability at $\mathrm{TeV}$ energies have been observed since its detection in 2006. GeV, X-ray, and radio emission have been detected along the entire orbit, enabling detailed study of the orbital modulation pattern and its super-orbital period.

Previously reported $\mathrm{TeV}$ baseline emission and spectral variations may indicate a neutron star flip-flop scenario, in which the binary system switches between accretor and propeller phases at different phases of the orbit.
\end{abstract}

Since September 2007, VERITAS has observed LS I +61 303 over three additional seasons, accruing $220+$ hours of data during different parts of its orbit. In this work, we present a summary of recent and long-term VERITAS observations of LS I +61 303. This analysis includes a discussion of the observed variation of $\mathrm{TeV}$ emission during different phases of the orbit, and during different superorbital phases.

36th International Cosmic Ray Conference -ICRC2019-

July 24th - August 1st, 2019

Madison, WI, U.S.A.

\footnotetext{
*Speaker

$\dagger$ http://veritas.sao.arizona.edu; for collaboration list see PoS(ICRC2019)1177.
} 


\section{Introduction}

The High Mass X-Ray Binary (HMXB) LS I +61॰ 303 was discovered by COS B in 1977 as an unidentified Galactic MeV gamma-ray emitter (2CG $135+01)$ [1]. 2CG $135+01$ was found to be positionally coincident with variable radio source GT $0236+610$, which exhibited strong non-thermal radio bursts [2]. Long term radio observations of GT 0236 exhibited a 26.496 day (orbital) periodicity with a possible 4 year-long (superorbital) modulation of the radio amplitude [3]. The COS B data failed to show the reported 26.496 day radio periodicity. Subsequently, observations of 2CG $135+01$ by COMPTEL between 1991 and 1994 revealed a persistent hard gamma-ray source, but with a factor of 10-100 reduced flux compared to the COS B detection [4]. COMPTEL did not find evidence for the 26.5 day modulation of the $>1 \mathrm{MeV}$ gamma-ray signal, confirming the lack of modulation observed by COS B. GT $0236+610$ was identified by VLBI observations in 1993 as a binary system with two components separated by $3.1 \times 10^{11}$ $(\mathrm{D} / 2.3 \mathrm{kpc}) \mathrm{m}$ [5], classifying it as a binary system. GT $0236+610$ was subsequently identified as associated with the optical source LS I $+61^{\circ} 303$, which was spectroscopically identified as a massive (10-15 solar mass) early Be star [6].

LS I $+61^{\circ} 303$ was also detected in X-rays [7, 8], exhibiting a hard X-ray spectrum consistent with other HMXB. LS I $+61^{\circ} 303$ was first identified as a periodic TeV emitter by the MAGIC collaboration in 2006 [9], and subsequent observations of LS I $+61^{\circ} 303$ by VERITAS demonstrated a combination of episodic flaring [10] with orbitally modulated $(26.496 \pm 0.0028$ day period) $\mathrm{TeV}$ emission [11]. The strongest $\mathrm{TeV}$ emission is consistently between phase range $\varphi$ $=0.55-0.65$ near apastron $(\varphi=0.7775)$. Longer-term modulation of the TeV emission, consistent with superorbital modulation (1667 \pm 8 day period) has also been demonstrated in a joint analysis of MAGIC and VERITAS observations [12]. In the GeV gamma-ray energies, LS I $+61^{\circ} 303$ demonstrated orbitally modulation with a $6 \mathrm{GeV}$ cutoff and strongest emission around $\varphi=0.3$, as reported by Fermi observations [13]. The strongest $\mathrm{GeV}$ emission is coincident with the periastron passage of the compact object around the Be star. Both X-ray [14] and GeV emission $(\mathrm{E}<5 \mathrm{GeV})$ are found to be superorbitally modulated, with a dip in the periastron $\mathrm{GeV}$ maximum near superorbital phase 0.4 , and a peak in the apastron $\mathrm{GeV}$ emission near superorbital phase $0.2[15]$.

Previous multiwavelength observations of LS I $+61^{\circ} 303$, using TeV observations by VERITAS and MAGIC combined with GeV observation by Fermi, and X-ray observation by SWIFT, have revealed conclusive evidence of a correlation between $\mathrm{X}$-ray and $\mathrm{TeV}$ emission during flaring states $[16,17,18]$, although earlier VERITAS studies failed to establish a significant correlation [19] . This correlation has been interpreted as requiring a common origin for both the XRay and TeV emission, providing evidence for an SSC or EC production mechanism. The multiwavelength observations also demonstrated an absence of any significant correlation between $\mathrm{TeV}$ emission and $\mathrm{GeV}$ emission. The lack of a correlation between $\mathrm{GeV}$ emission and $\mathrm{X}$ $\mathrm{ray} / \mathrm{TeV}$ emission has been interpreted as requiring a second, independent emission mechanism for the $\mathrm{GeV}$ emission.

An astrophysical model of LS I $+61 \circ 303$ continues to emerge based upon the above (and other) multiwavelength observations [20]. The current model envisions a rapidly spinning Be star, spectral type B0Ve in a binary association with a compact object in an eccentric orbit $(\mathrm{e}=0.54$ \pm 0.03 ) around a giant Be type 10-15 solar mass star. The Be star is thought to possesses a strong wind and a circumstellar decretion disk which crosses the plane of the orbit in two places, 
near orbital periastron [21]. The mass of the compact object is not completely determined, and arguments have been made for either a neutron star (NS) or a black hole with precessing jets. $\mathrm{TeV}$ gamma rays are produced by either the shock front of the colliding pulsar and Be star's winds, or by the relativistic precessing jets which are powered by accreting matter from the Be star onto the blackhole. More comprehensive models, such as the Ejector-Propellor model [22], which explores the effects of different radiative and wind pressures between apastron and periastron to explain the observed differences between the orbital $\mathrm{GeV}$ and $\mathrm{TeV}$ modulations. However, no current model adequately explains all the observed multiwavelength features of this system.

\section{The VERITAS IACT Observatory}

The VERITAS Observatory [23] is an array of 4 Imaging Atmospheric Cherenkov Telescopes (IACTs) located at the F.L. Whipple Observatory, Arizona, USA $\left(31^{\circ} 40 \mathrm{~N}, 110^{\circ} 57 \mathrm{~W}, 11268\right.$ m.a.s.l). Each telescope features an f/1.0 $12 \mathrm{~m}$-diameter Davies-Cotton reflector consisting of 345 hexagonal facets, resulting in $110 \mathrm{~m}^{2}$ of light collecting area. The separation between nearest neighbor telescopes is currently $80-120 \mathrm{~m}$. The Davis-Cotton reflector provides a 4 nanosecond spread to photons arriving at the focal plane. The camera is pixelated with 499 photo-multiplier tubes (PMTs) each of $28.6 \mathrm{~mm}$ diameter. The PMTs are UV sensitive and have fast rise times coupled with high quantum efficiency. VERITAS has been upgraded twice: In 2009, Telescope 1 (T1) was relocated to a more symmetrical position/longer baseline in the array to increase sensitivity by $30 \%$ [24]. The second upgrade (2012) upgraded the photomultiplier tubes (PMTs) in each camera to increase average peak quantum efficiency from $20 \%$ at $320 \mathrm{~nm}$ to $>32 \%$ at $330 \mathrm{~nm}$ [25]. This second upgrade also upgraded the Level 2 (camera level pixel trigger pattern) triggering system to improve trigger timing alignment, reduce L2 time coincident window, and increases rejection of random PMT triggered events [26]. The net effect of these two upgrades reduced VERITAS's gamma-ray threshold from $120 \mathrm{GeV}$ down to $85 \mathrm{GeV}$, and an increase in VERITAS gamma-ray detection rate by $50 \%$ [27].

In its current configuration, VERITAS is sensitive to VHE gamma rays with energies between $85 \mathrm{GeV}$ to $>30 \mathrm{TeV}$ [28]. VERITAS nominally detects a source with $1 \%$ flux of the Crab Nebula in less than $25 \mathrm{~h}$ of observation.

\section{VERITAS LSI +61303 Observations (2007-2016)}

VERITAS started observing LS I $+61^{\circ} 303$ in 2006 and has continued its campaign every year, accumulating more than $200 \mathrm{~h}$ of observations up to December 2016. Previous LS I $+61^{\circ} 303$ results used data collected between October 5, 2007 and November 23, 2016 covering three different epochs of the telescope operations [29]. Observations were performed in wobble mode with $0.5^{\circ}$ offset, a standard mode for VERITAS observations. The combination of VERITAS latitude and source declination causes LS I $+61^{\circ} 303$ to transit the sky at medium elevations; observations are within in $29^{\circ}-42^{\circ}$ zenith. The entire dataset covers all orbital phases, with maximum exposures around apastron. The observations are quality-selected to remove minutes affected due to weather and hardware problems. Standard analysis for VERITAS point sources, using Event Display and VEGAS analysis packages, were performed on the data. Gamma-ray selection techniques used included using both box cuts and Boosted Decision Tree (BDT) algorithms [30]. After applying standard gamma-selection cuts, the observations have an effective gamma-ray threshold $\mathrm{E}_{\text {thresh }}>300 \mathrm{GeV}$. 
Using the zero orbital phase $\varphi_{0}=$ MJD 43366.775 and an orbital period $P_{o r b}=26.4960$ days, the data set is divided into 10 phases with width $\Delta \varphi=0.1$. Except for three individual seasons $(2008 / 2009,2009 / 2010$, and 2010/2011), the source is detected every season with significance varying between $5.6 \sigma$ and $21 \sigma$, generally near its apastron passage of the orbit. Observations performed during these ten years also demonstrate evidence for low-level persistent $\mathrm{TeV}$ emission ( 3\% Crab flux) [29].

$\mathrm{TeV}$ outbursts are generally observed from LS I +61 o 303 close to its apastron passage in the phase range $\varphi=0.6-0.7$ when flux observed can be well above $15 \%$ of the Crab Nebula. Photons with energy of $10 \mathrm{TeV}$ or more have been observed during these flaring states implying the existence of energetic particles with tens of $\mathrm{TeV}$ energy in the binary system. During this period, there was a single episode of significant emission (5.6 $)$ from LS I $+61^{\circ} 303$ during its superior conjunction, closer to its periastron passage [10].

\section{VERITAS LSI +61 303 Observations (2016-2019)}

During 2016-2019, VERITAS recorded an additional 32.7 hours of observations on LS I +61。 303 after weather and data quality cuts. The exposures in different years are distributed unevenly in various phase bins due to the full moon and other observing constraints. During 20162017 and 2018-2019, VERITAS observations primarily covered phases 0.4 to 0.9 . These phases are near apastron, where the greatest $\mathrm{TeV}$ emission is traditionally observed. Observations in 2017-2018 covered phases near periastron ( 0.0 to 0.4 ) where $\mathrm{TeV}$ emission has generally been suppressed (Figure 1).

\section{VERITAS exposure 2016-2019}

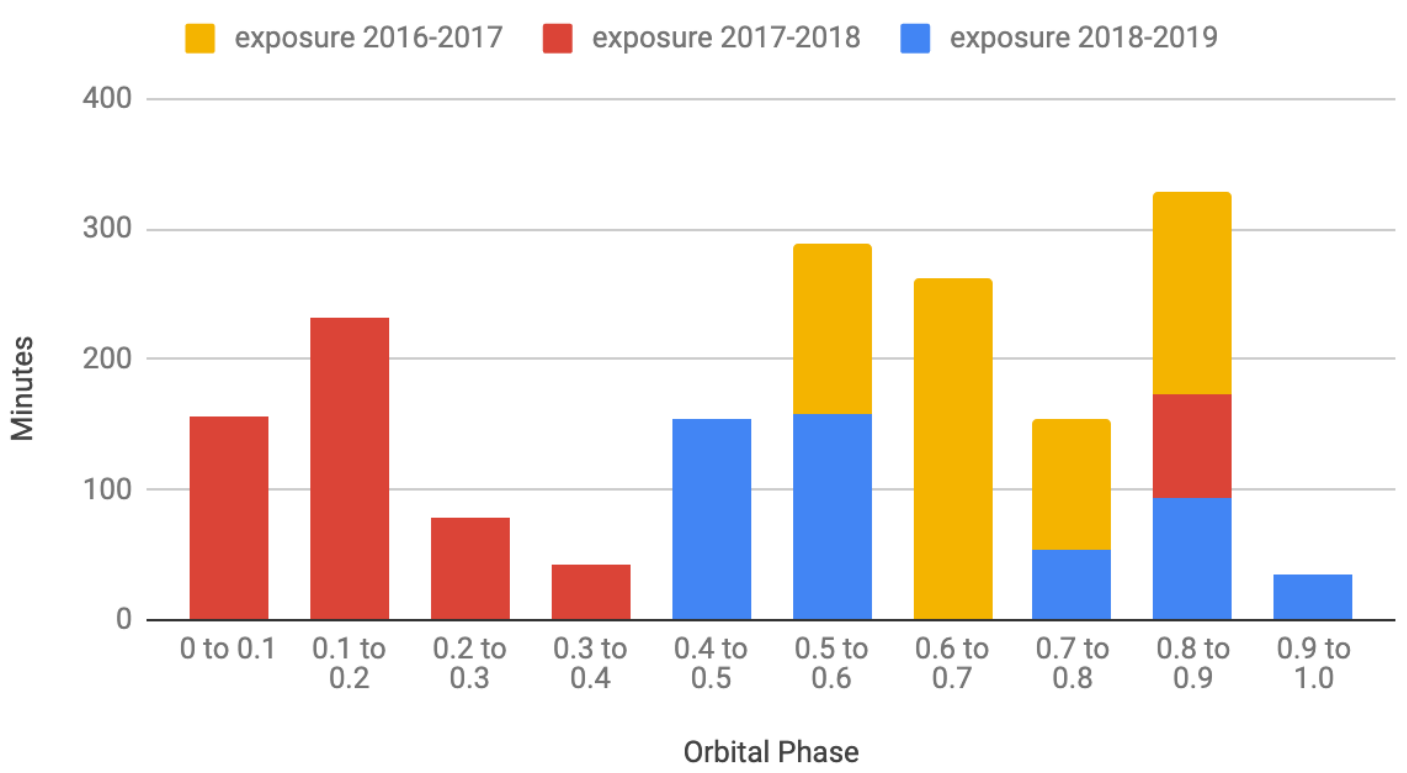

Figure 1: VERITAS exposure on LS I +61 303 during 2016-2019, broken down according to orbital phase and histogrammed according to individual years. Yellow: 2016-2017 exposure. Red: 2017-2018 exposure. Blue: 2018-2019 exposure. 
Figure 2 shows the combined exposure of VERITAS LS I $+61^{\circ} 303$ observations during the period 2007-2019, which spans three observing seasons (2016-2017, 2017-2018, and 2018-2019). During this period, more than 220 hours of data are available after weather and data quality cuts. Table 1 provides a detailed summary of the analysis results for each observing season. The table lists of the MJD range of the observations, the observed statistical significance $\sigma$ and normalized statistical significance $\sigma / \sqrt{\text { hours }}$, and the range of orbital phases and superorbital phases observed during each observing season. When integrating over each observing season, we observe a statistically strong detection $(11.37 \sigma)$ of LS I $+61^{\circ} 303$ only during 2016-2017, when both orbital phase range is near apastron. During this season, the superorbital phase $(0.5)$ is also considered to be favorable, if previously report superorbital modulation trend [14] continues unabated. During 2017-2018 we observe only a marginal excess $(2.72 \sigma)$ for the seasonal statistical significance as expected during LS I $+61^{\circ} 303^{\prime}$ 's periastron passage. We also note that the superorbital phase of this season (0.8) predicts suppressed $\mathrm{TeV}$ emission.

For observing season 2018-2019, only a marginal excess $(3.48 \sigma)$ is observed for LS I $+61^{\circ} 303$ even though the observations were made around apastron, identical to 2016-2017. The 20182019 LS I $+61^{\circ} 303$ observations were made at superorbital phase 0.0 , which is predicted to coincide with the minimum $\mathrm{TeV}$ emission due to superorbital modulation. The seasonal VERITAS observations of LS I $+61^{\circ} 303$ during the past three years are consistent with a continuation of the previously reported superorbital modulation of $\mathrm{TeV}$ emission[14].

At the conference, we expect to show an updated LS I $+61^{\circ} 303$ analysis from the combined period 2007-2019.

\section{7-2019 total exposure}

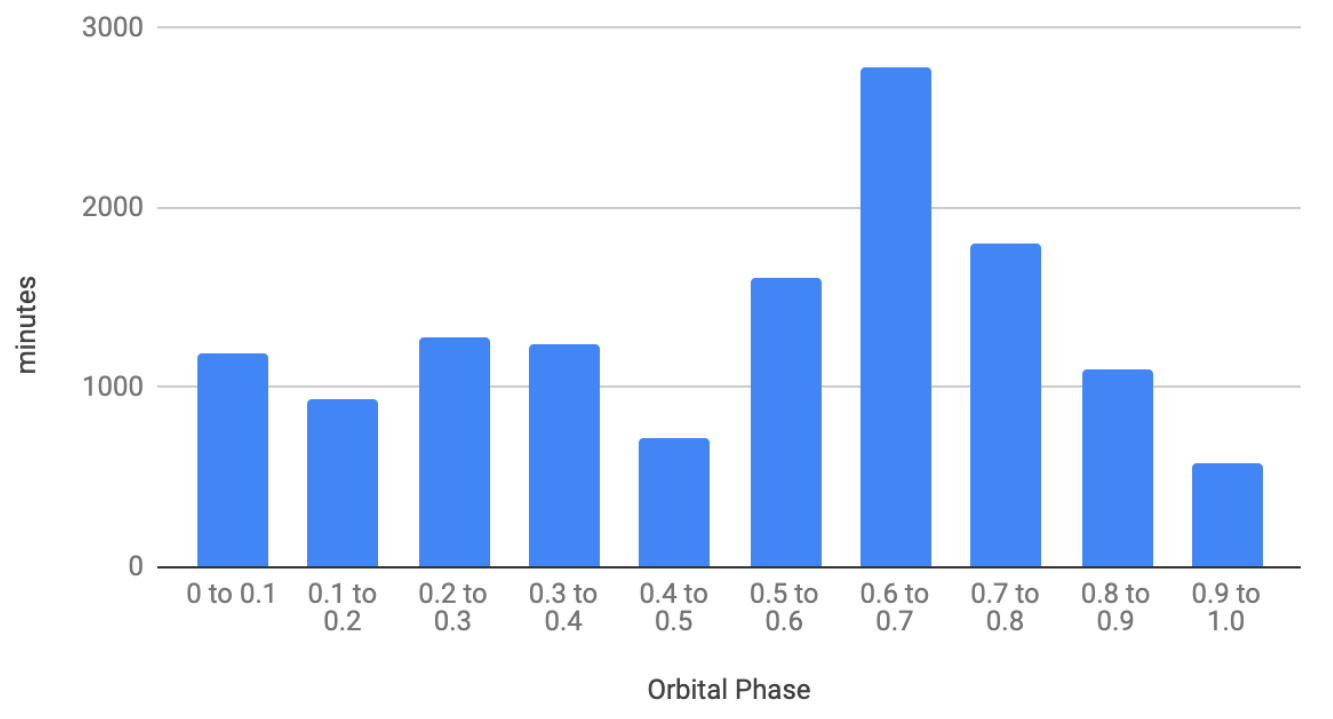

Figure 2: Cumulative VERITAS exposure on LS I +61 303 during 2007-2019, broken down according to the orbital phase. 
Table 1: Summary of VERITAS seasonal observations of LSI $+61^{\circ} 303$. The right two columns indicate the orbital and supraorbital phase ranges for the corresponding observation date range. Green cells: Observations are within favorable phase ranges for strong emission. Red cells: Observations are within unfavorable phase range for strong emission. Observations within one or more unfavorable phase ranges are expected to have suppressed TeV gamma-ray emission.

\begin{tabular}{|c|c|c|c|c|c|}
\hline $\begin{array}{c}\text { Observing } \\
\text { Season }\end{array}$ & $\begin{array}{c}\text { MJD } \\
\text { Observation } \\
\text { Date Range }\end{array}$ & $\begin{array}{c}\text { Total } \\
\text { Significance } \\
\sigma\end{array}$ & $\begin{array}{c}\text { Normalized. } \\
\text { Significance } \\
\sigma / \sqrt{\text { hours }}\end{array}$ & $\begin{array}{c}\text { Orbital } \\
\text { Phase } \\
\text { Range }\end{array}$ & $\begin{array}{c}\text { Superorbital } \\
\text { Phase }\end{array}$ \\
\hline $2016-2017$ & $57662-57696$ & 11.37 & 3.32 & $0.5-0.8$ & 0.5 \\
\hline $2017-2018$ & $58028-58051$ & 2.72 & 0.77 & $0.8-1.4$ & 0.8 \\
\hline $2018-2019$ & $58402-58492$ & 3.48 & 1.18 & $0.4-0.9$ & 0.0 \\
\hline
\end{tabular}

\section{Acknowledgments}

This research is supported by grants from the U.S. Department of Energy Office of Science, the U.S. National Science Foundation and the Smithsonian Institution, and by NSERC in Canada. This research used resources provided by the Open Science Grid, which is supported by the National Science Foundation and the U.S. Department of Energy's Office of Science, and resources of the National Energy Research Scientific Computing Center (NERSC), a U.S. Department of Energy Office of Science User Facility operated under Contract No. DE-AC02-05CH11231. We acknowledge the excellent work of the technical support staff at the Fred Lawrence Whipple Observatory and at the collaborating institutions in the construction and operation of the instrument.

\section{References}

[1] W. Hermsen et al., New high energy $\gamma$-ray sources observed by COS B, Nature 269, 494 October 1977.

[2] P.C. Gregory \& A.R. Taylor, New highly variable radio source, possible counterpart of $\gamma$-ray source CG135+1, Nature 272, 7041978.

[3] A. R. Taylor \& P.C. Gregory, Periodic Radio Emission from LS I +61 303. Ap J. 255, 210-216. 1982.

[4] R. van Dijk et al., COMPTEL detection of the high-energy $\gamma$-ray source $2 C G 135+01, A \& A \mathbf{3 1 5}$, 485-492 November 1996.

[5] M. Massi et al., High resolution radio map of the X-ray binary LSI+61 303, A\&A 269, 249 1993.

[6] J.B. Hutchings and D. Crampton, Spectroscopy of the unique degenerate binary star LSI +61303 , PASP 93, 486 August 1981.

[7] G.F. Bignami G.F. et al. , Einstein X-ray identification of the variable radio star LS I +61 303, ApJ 247, L85 July 1981.

[8] P. Goldoni and S. Mereghetti, X-ray observations of the peculiar Be star LSI +61 303, A\&A 299, 751 July 1995. 
[9] Albert J. et al., Periodic Very High Energy $\gamma$-Ray Emission from LS I $+61^{\circ} 303$ Observed with the MAGIC Telescope, ApJ, 693, 303 March 2009.

[10] S. Archambault and et al. (VERITAS Collaboration), Exceptionally Bright TeV Flares from the Binary LS I +61 303, ApJ 817, L7 January 2016.

[11] V. A. Acciari and et al. (VERITAS Collaboration), VERITAS Observations of the $\gamma$-Ray Binary LS I +61 303, ApJ 679, 1427-1432 June 2008.

[12] M. L. Ahnen and et al. (MAGIC Collaboration), Super-orbital variability of LS I+61 303 at TeV energies, $A \& A$ 591, A76 June 2016.

[13] A.A. Abdo et al., Fermi-LAT Observations of LS I +61 303: First Detection of an Orbital Modulation in GeV Gamma Rays, ApJ, 701, L123 August 2009.

[14] M. Chernyakova, A. Neronov, S. Molkov, D. Malyshev, A. Lutovinov and G. Pooley, Superorbital Modulation of X-Ray Emission from Gamma-Ray Binary LSI+61 303, ApJ 747, L29 March 2012.

[15] Y. Xing, Z. Wang and J. Takata, Superorbital Modulation at GeV Energies in the $\gamma$-Ray Binary LS $I+61^{\circ} 303$, ApJ 851, 92 December 2017.

[16] H. Anderhub, L. A. Antonelli, P. Antoranz, M. Backes, C. Baixeras, S. Balestra, et al., Correlated XRay and Very High Energy Emission in the Gamma-Ray Binary LS I +61 303, ApJ 706, L27 November 2009.

[17] P. Kar and VERITAS Collaboration, Long-term VERITAS monitoring of LS I $61+303$ in conjunction with X-ray, and GeV observation campaigns, in 34th International Cosmic Ray Conference (ICRC2015), vol. 34 of International Cosmic Ray Conference, p. 818, July, 2015

[18] P.Kar, High Mass X-ray Binaries at Extreme Energies with VERITAS, PhD Thesis, University of Utah, December 2017.

[19] A. Aliu et. al, Multiwavelength Observations of the TeV Binary LS I $+61^{\circ} 303$ with VERITAS, Fermi-LAT, and Swift/XRT during a TeV Outburst, ApJ 779, 88 November 2013.

[20] B. Marcote, Review on the multiwavelength emission of the gamma-ray binary LS I +61303 , PoS(MULTIF2017) 045 June 2017.

[21] R. K. Zamanov et al., Optical spectroscopy of Be/gamma-ray binaries, $A \& A$ 593, A97 September 2016.

[22] R. K. Zamanov, An Ejector-Propeller Model for LSI+61 303. Mon. Not. R. Astron. Soc. 272, 308 January 1995.

[23] Holder, J., et al. The First VERITAS Telescope. Astropart.Phys. 25, 391-401, 2006

[24] Perkins, J., et al. VERITAS Telescope 1 Relocation: Details and Improvements. 2009 Fermi proceedings, arXiv:0912.3841

[25] Otte, N. et al. The Upgrade of VERITAS with High Efficiency Photomultipliers. 2011 ICRC proceedings, arXiv:1110.4702. July 2011.

[26] B. Zitzer et al., The VERITAS Upgraded Telescope-Level Trigger Systems: Technical Details and Performance Characterization. 2013 ICRC proceedings, arXiv:1307.8360. July 2013. 
[27] D. B. Kieda et al., The Gamma Ray Detection sensitivity of the upgraded VERITAS Observatory, 2013 ICRC proceedings arXiv:1308.4849 July 2013.

[28] N. Park and VERITAS Collaboration, Performance of the VERITAS experiment, in 34th International Cosmic Ray Conference (ICRC2015), vol. 34 of International Cosmic Ray Conference, p. 771, July, 2015.

[29] P. Kar et al., Decadal VERITAS Observation of LS I +61 oo303: Detection of TeV emission around the entire orbit, PoS(ICRC2017) 712.

[30] M. Krause, E. Pueschel, and G. Maier, Improved $\gamma /$ hadron separation for the detection of faint y-ray sources using boosted decision trees, Astroparticle Physics 89, 1 March 2017. 